\title{
La paradoja de la autoridad: el positivismo jurídico y el fundamento del derecho
}

\author{
The Possibility Puzzle: Legal Positivism \\ and the Ground of Law
}

Francesca Poggi*

Recepción y evaluación de propuesta: 24/10/2017

Aceptación: 26/02/2018

Recepción y aceptación final: 16/08/2018

\begin{abstract}
Resumen: El objetivo de este ensayo es discutir una paradoja sobre los orígenes del derecho, que Shapiro analiza ampliamente en su libro, señalándola como una prueba clave para toda la teoría jurídica: la Paradoja de la Autoridad (PA). En resumen, por un lado, alguien tiene el poder de crear normas jurídicas solo si una norma existente le confiere ese poder, pero, por otro lado, una norma que confiere el poder de crear normas jurídicas solo existe si alguien que tiene el poder de crearla la crea. En este trabajo voy a tratar de mostrar (i) que una vez que adoptamos algunas distinciones, la paradoja de Shapiro se desvanece; (ii) que todos los positivistas jurídicos solucionan de la misma manera la paradoja en cuestión, y que esto se sigue directamente de la definición de derecho ofrecida por el positivismo jurídico; (iii) que todos los problemas, conectados con la paradoja y antes mencionados, han sido ya resueltos por la teoría iuspositivista del derecho.
\end{abstract}

Profesora, Dipartimento Cesare Beccaria, Università degli Studi di Milano, Italia. E-mail: francesca.poggi@unimi.it. Quiero agradecer a todos los participantes y organizadores del seminario sobre el libro de Scott Shapiro, Legality, por sus observaciones y sugerencias. Quiero agradecer también a Diego dei Vecchi y Federico José Arena tanto por la ayuda prestada en la traducción de este texto al español, como por sus agudos comentarios. 
Palabras clave: paradoja de la autoridad, positivismo jurídico, regla de reconocimiento, poder.

\begin{abstract}
The aim of this essay is to discuss a puzzle concerning the law, which Shapiro analyses in his book. Briefly, the problem is that in order to get legal power, one must already have legal power. In this essay I will sustain, firstly, that once we adopt a legal positivist point of view, the puzzle vanishes or, better, it turns into a not puzzling question; secondly, that all the legal positivists (including Hart and Austin) give the same answer to that question, and this because that answer stems from (is implicit in) the very concept of legal positivism; finally, that the underlying problems (previously mentioned) had already been solved by a legal positivist theory. With regard to the last point, I will try to vindicate Hart's theory against Shapiro's criticisms, although I will acknowledge that some corrections must be made.
\end{abstract}

Keywords: possibility puzzle, legal positivism, rule of recognition, power.

\title{
I. La paradoja de la autoridad
}

El objetivo de este ensayo es discutir una paradoja sobre los orígenes del derecho, que Shapiro señala y analiza ampliamente en su libro. Mi tarea no es simple, ya que la paradoja de Shapiro está íntimamente relacionada con problemas muy complejos y muy controvertidos en la filosofía del derecho, como la metodología de la teoría jurídica, el estatus lógico de los enunciados normativos, el deber judicial de aplicar el derecho, y la relación entre deberes morales y deberes jurídicos. El libro de Shapiro tiene sin dudas el mérito de haber señalado la importancia de esta paradoja como una prueba clave para toda la teoría jurídica. Sin embargo, en este trabajo voy a tratar de mostrar (i) que una vez que adoptamos algunas distinciones analíticas, la paradoja de Shapiro se desvanece o, mejor dicho, resulta no ser paradójica en absoluto; (ii) que todos los positivistas jurídicos solucionan de la misma manera la paradoja en cuestión, y que esto no podría ser de otro modo en tanto que tal solución se sigue directamente de la definición de derecho ofrecida por el positivismo jurídico; (iii) que todos los problemas, conectados con la paradoja y antes mencionados, han sido ya resueltos por la teoría 
La paradoja de la autoridad: el positivismo jurídico...

iuspositivista del derecho. Con respecto al último punto, voy a tratar de reivindicar la teoría de Hart frente a las críticas de Shapiro, aunque reconozco que son necesarias algunas correcciones.

Según Shapiro, cualquier intento de explicar cómo el derecho ha sido inventado se enfrenta a una paradoja, que denomina Paradoja de la Autoridad (PA). Esta paradoja puede resumirse de la siguiente manera:

Huevo: Un sujeto está facultado para crear normas jurídicas únicamente si una norma existente le confiere esa potestad.

Gallina: Una norma que faculta a crear normas jurídicas existe únicamente si un sujeto con la potestad para hacerlo la ha creado $^{1}$.

En suma, el problema es que "a fin de adquirir potestad jurídica, uno debe ya tener potestad jurídica" ${ }^{2}$. En otras palabras: ¿qué fue primero, el huevo o la gallina?

Shapiro señala que los iusnaturalistas y los positivistas jurídicos proponen soluciones diferentes a la PA.

Los teóricos (contemporáneos) del derecho natural piensan que el derecho no está determinado solo por hechos sociales, sino que la autoridad jurídica debe fundarse, en última instancia, en normas morales. En suma, los iusnaturalistas (contemporáneos) eligen la gallina y rechazan el huevo: creen que hay normas (normas morales, que pertenecen a la ley natural) que confieren el poder de crear normas jurídicas y que, sin embargo, no fueron ellas mismas creadas por alguien autorizado por otras normas, o, mejor dicho, que no fueron creadas en absoluto.

Por el contrario, los positivistas jurídicos afirman que el derecho es, en última instancia, determinado solo por hechos sociales. Según Shapiro, las soluciones iuspositivistas a la PA no son homogéneas.

1 Shapiro, S., Legalidad, Madrid-Barcelona, Marcial Pons, 2014, p. 70.

2 Ibidem, p. 67. 
Shapiro analiza dos de estas soluciones: la solución de Austin y la de Hart. En el apartado siguiente voy a examinar cómo Shapiro reconstruye las soluciones ofrecidas por estos dos autores y cuáles son las críticas que les dirige.

\section{I.2. Las soluciones de Austin y Hart (según Shapiro)}

Según Shapiro, Austin rechaza el huevo (y elige la gallina) en cuanto sostiene que el poder de crear normas jurídicas no (siempre) tiene que ser otorgado por otras normas. Según Austin, la soberanía se basa en los hábitos de obediencia (el soberano es una persona, o un conjunto determinado de personas, que es habitualmente obedecido y que no obedece a nadie), de modo que no hay necesidad de postular una norma que confiera el poder soberano.

Shapiro, de acuerdo con Hart, sostiene que la solución de Austin no puede explicar tres características esenciales de todo sistema jurídico: la continuidad, la persistencia y la limitación ${ }^{3}$. Por otra parte, Shapiro cree que el intento de Austin de cumplir con la Ley de Hume no fue exitoso. Austin trató de evitar la deducción de un 'deber ser' de un 'ser' configurando los conceptos jurídicos, y los enunciados y los juicios de valor en que ocurren, como descriptivos. Así, siendo descriptivos, y no normativos, los juicios sobre deberes y derechos podrían derivarse de enunciados sobre hábitos de obediencia y probabilidades, que tienen a su vez un carácter meramente descriptivo. En otras palabras, Shapiro sostiene que, de acuerdo con Austin, el siguiente razonamiento es meramente descriptivo (en términos de Shapiro, es un patrón DEDS):

(i) Si el soberano ha promulgado la norma primaria 'Todos deben hacer $p$ ' y la norma secundaria 'Quién no hace $p$ debe ser castigado con la sanción $S$ ', entonces,

(ii) Todos deben (tienen la obligación de) hacer $p$ 
La paradoja de la autoridad: el positivismo jurídico...

La premisa (i) y la conclusión (ii) son descriptivas y, según Austin, son idénticas a:

(i) Si el que es habitualmente obedecido y que no obedece a ninguna otra persona expresa su voluntad de que todos deben hacer $\mathrm{p}$ y expresa también su voluntad de que quien no hace $\mathrm{p}$ sea castigado con la sanción $\mathrm{S}$, entonces

(ii) Es probable que, si alguien no hace $\mathrm{p}$, sea castigado con la sanción $\mathrm{S}$

Sin embargo, según Shapiro,

[es] difícil de ver cómo los enunciados que emplean conceptos puramente descriptivos podrían ser utilizados en su forma normal, a saber, para justificar y evaluar. Cuando decimos a otros que están obligados a realizar alguna acción, intentamos expresar una razón para que lo hagan. De igual forma, cuando criticamos a otros por violar sus obligaciones, estamos presuponiendo que debían haber actuado de manera diferente. Decimos que han actuado "de manera incorrecta" y que son "culpables" de una "infracción". Si algunos conceptos son normativos, estos lo son".

En realidad, me parece que la teoría de Austin puede explicar perfectamente cómo los enunciados sobre obligaciones y deberes, a pesar de ser puramente descriptivos, pueden ser empleados en justificaciones y evaluaciones. Si el soberano ha promulgado la norma primaria 'Todos deben hacer $p$ ' y la norma secundaria 'Quién no hace $p$ debe ser castigado con la sanción $S$ ', esta es una buena razón para hacer $p$. En otras palabras, la proposición descriptiva 'Si no hace $p$, es probable que sea castigado' funda la norma técnica (la directiva, en términos de von Wright) 'Si desea evitar la probabilidad de ser castigado, usted debe hacer $p$ '. La razón para hacer $p$ es que no se desea ser (probablemente) castigado. El deseo de no ser castigados es una muy buena razón para hacer varias cosas. De la misma manera, los enunciados descriptivos de Austin se pueden 
emplear para evaluar: se puede criticar a alguien porque ha hecho algo por lo cual será castigado. Sin embargo, es evidente que, según Shapiro, estos enunciados y estos conceptos a veces tienen un significado diferente. A veces, según Shapiro, al afirmar que alguien debe hacer $p$, o que no hacer $p$ está mal, queremos expresar normas, juicios prescriptivos. Pero esto no significa que la teoría de Austin no cumpla con la ley de Hume; solo significa que no se corresponde con nuestras intuiciones acerca de la función de los enunciados sobre deberes y derechos.

Según Shapiro, la estrategia de Hart para resolver la PA consiste, en cambio, en rechazar la gallina: Hart afirma que, mientras que los poderes deben ser otorgados por normas, las normas pueden ser creadas por alguien que no tenía la autoridad para hacerlo. De hecho, de acuerdo con Hart,

[1]a afirmación de que ella existe sólo puede ser un enunciado de hecho externo. Porque mientras que una regla subordinada de un sistema puede ser válida y, en ese sentido, "existir" aún cuando sea generalmente desobedecida, la regla de reconocimiento sólo existe como una práctica compleja, pero normalmente concordante, de los tribunales, funcionarios y particulares, al identificar el derecho por referencia a ciertos criterios. Su existencia es una cuestión de hecho ${ }^{5}$.

Las críticas de Shapiro contra Hart consisten en sostener que las normas sociales no pueden reducirse a prácticas sociales ni son necesariamente generadas por prácticas sociales. De hecho, Shapiro sostiene que, de acuerdo con Hart,

Para crear una regla social, los individuos no tienen que tener autoridad; en lugar de ello, solo se requiere que participen en

5 Hart, H. L. A. (1961), The Concept of Law, Second Edition, Oxford, Claredon Press, 1994, p. 110 (137). [N. E.: citado en la traducción de la primera edición de Genaro Carrió: Hart, H. L. A., El concepto de derecho, Buenos Aires, Abeledo-Perrot, 1963. En paréntesis se indican las páginas correspondientes a la traducción]. 
La paradoja de la autoridad: el positivismo jurídico...

una práctica social. Ello en tanto la teoría de las practicas sociales entiende que las reglas sociales son solo prácticas sociales ${ }^{6}$.

Shapiro objeta que las normas sociales no pueden reducirse a las prácticas sociales ya que normas y prácticas pertenecen a diferentes categorías metafísicas. Por otra parte, Shapiro afirma que incluso la tesis más débil, según la cual las normas sociales son generadas por prácticas sociales, no funciona: muchas de nuestras prácticas sociales no generan regla social alguna. Shapiro muestra este punto a través del siguiente ejemplo:

Entre los miembros de la clase profesional en Estados Unidos, se acepta en general que no se debe fumar incluso cuando nadie más resulte afectado. Los fumadores son cotidianamente criticados por los no-fumadores. Fumar, dicen, es "una tontería", un "hábito desagradable" y da un "mal ejemplo". Además, estos no-fumadores no son criticados por otros no-fumadores por realizar tales críticas. Sin embargo, no hay regla social en contra de fumar solo o con otros fumadores ${ }^{7}$.

Creo que estas críticas a Hart no son convincentes.

En primer lugar, no estoy segura de que Hart identifique normas sociales con prácticas sociales. Como escribe Shapiro: "las reglas sociales son creadas simplemente en virtud de que son aceptadas y prac-

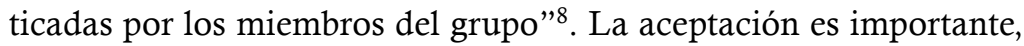
así como la práctica y, si existe una diferencia metafísica fundamental entre prácticas y normas, no estoy tan segura de que tal diferencia fundamental exista también entre normas y actitudes de aceptación.

De todos modos, el punto fundamental es que, como se puede deducir también de la cita de Shapiro, la práctica y la actitud de aceptación no son ellas mismas normas sociales, sino condiciones de existencia de normas sociales. Para citar a Hart, "para que exista una regla social por lo menos algunos tienen que ver en la conducta de que se trata una

6 Shapiro, S., op. cit., p. 133.

7 Ibid, p. 143.

8 Ibid, p. 133. 
pauta o criterio general de comportamiento a ser seguido por el grupo como un todo", y "en la existencia de reglas sociales está de por medio una combinación de conducta regular con una actitud distintiva hacia esa conducta en cuanto pauta o modelo de comportamiento"10. Por lo tanto, me parece que Hart adopta la tesis débil: la que afirma que las normas sociales son generadas por las prácticas sociales. Como hemos visto, Shapiro objeta que no todas las prácticas sociales, aceptadas como estandardes generales, generan normas sociales. ¿Es esta tesis correcta? ¿No existe realmente una regla social contra el tabaquismo dentro de la clase profesional en los Estados Unidos? Por supuesto que no existe una prohibición jurídica de fumar (en una casa particular o al aire libre), pero ¿qué pasa con otros tipos de normas sociales? Ciertamente no decimos que, entre la clase profesional en los Estados Unidos, existe una obligación de no fumar, pero de acuerdo con Hart,

no siempre es el caso que cuando existen reglas, la conducta requerida por ellas es concebida en términos de obligación [...] Se dice y se piensa que una regla impone obligaciones cuando la exigencia general en favor de la conformidad es insistente, y la presión social ejercida sobre quienes se desvían o amenazan con hacerlo es grande ${ }^{11}$.

Podemos pensar que dentro de la clase profesional en Estados Unidos existe una regla social contra el tabaquismo (una regla que pertenece a la moral de ese grupo social), pero que la presión social y la demanda de conformidad no son (aún) lo suficientemente generales y fuertes como para que esta regla sea concebida en términos de obligación. Se trata de una cuestión de grado: una respuesta clara no es posible. En otras palabras, aquí nos preguntamos si existe una norma consuetudinaria contra el tabaquismo. La existencia de normas consuetudinarias es una cuestión muy compleja. Tan compleja que, para

9 Hart, H. L. A., op. cit., p. 56 (71), énfasis agregado.

${ }^{10} \mathrm{Ibid}$, p. 85 (106), énfasis agregado.

11 Ibidem, pp. 85-86 (107). 
La paradoja de la autoridad: el positivismo jurídico...

resolverla, en la comunidad primitiva imaginada por Hart fue necesario introducir la regla de reconocimiento.

Por último, me parece que para refutar la solución de Hart a la PA, no es ni necesario ni suficiente demostrar que algunas prácticas sociales no producen reglas sociales. Lo que hay que demostrar es, en cambio, que la práctica concordante de los tribunales, los funcionarios y las personas privadas en la identificación del derecho no puede producir una norma social. Es decir, hay que demostrar que la regla de reconocimiento no puede considerarse existente por el simple hecho de ser aceptada y practicada por los miembros de un grupo.

Shapiro dirige otra objeción a Hart. Según Shapiro, la solución de Hart al desafío de Hume queda seriamente indeterminada.

Prima facie, el concepto de punto de vista interno permite derivar un juicio normativo a partir de un mero hecho, en este caso, del hecho que existe la regla de reconocimiento. Como Shapiro señala:

Puede adoptarse el punto de vista interno hacia la práctica y tratar el patrón de comportamiento como estándar para la guía y la evaluación de la conducta. Y una vez se forma un juicio normativo respecto de la corrección de obedecer la regla de reconocimiento, pueden derivarse otros juicios normativos sobre la validez jurídica, los derechos y las obligaciones ${ }^{12}$.

Hart llama a estos juicio 'internos'. Consideremos los dos silogismos siguientes, HS1 (silogismo de Hart 1) y HS2 (silogismo de Hart 2)

HS1

Si la regla de reconocimiento establece que los jueces deben aplicar las normas que cumplen los criterios de validez $\mathrm{C}_{1}, \mathrm{C}_{2}$, $\mathrm{C}_{3}$, etc.

Si la regla 'Todos deben hacer $p$ ' cumple los criterios de validez $\mathrm{C}_{1}, \mathrm{C}_{2}, \mathrm{C}_{3}$, etc.

Si los jueces aceptan la regla de reconocimiento, entonces 
Los jueces deben aplicar la regla 'Todos deben hacer $p$ '

HS2

Si la regla 'Todos deben hacer $p$ ' existe

Si acepto la regla 'Todos deben hacer $p$ ', entonces

Todos (incluido yo) deben hacer $p$

HS1 es un silogismo judicial: su conclusión, que deriva de la aceptación de la regla de reconocimiento, no es que 'Todos deben hacer $p$ ', sino que los jueces (incluido yo, si soy un juez) deben aplicar la regla 'Todos deben hacer $p$ '. HS2, en cambio, es un silogismo del hombre común: su conclusión se deriva de aceptar no la regla de reconocimiento, sino la norma primaria 'Todos deben hacer $p$ '. Estos dos silogismos son válidos: por lo tanto parece que, si bien la existencia de una norma es un mero hecho, Hart no infringe la ley de Hume.

Sin embargo, según Shapiro, los juicios normativos se pueden emitir sin asumir el punto de vista interno: incluso cuando las personas no aceptan el derecho, es posible para ellas describir las normas jurídicas empleando una terminología normativa.

El hombre malo no acepta estas normas [las reglas secundarias del sistema], pero de todos modos puede redescribir adecuadamente el derecho en términos de obligaciones, derechos y validez jurídica ${ }^{13}$.

Esta objeción es problemática: no queda claro por qué la redescripción del derecho (como la etiqueta Shapiro) -el hecho de que un hombre malo, que toma el punto de vista externo, pueda redescribir la ley utilizando la terminología normativa-comprometa seriamente el cumplimiento de la ley de Hume por parte de Hart.

En primer lugar, de acuerdo con Hart, el punto de vista interno se manifiesta con típicas expresiones lingüísticas en el sentido de que si se acepta una norma como estándar general de conducta, entonces se pueden expresar juicios normativos empleando una terminología 
La paradoja de la autoridad: el positivismo jurídico...

típicamente normativa ('debería', 'debe', etc.) ${ }^{14}$. Sin embargo, esto no implica (desde el punto de vista lógico) que quien, mediante el uso de una terminología típicamente normativa, exprese juicios normativos, necesariamente acepte las normas. Afirmar lo contrario constituiría una instancia de la falacia lógica de la afirmación del consecuente. Se puede decir públicamente que todos deben pagar los impuestos, aun si no se los paga e incluso si se considera que nadie debería pagarlos. La hipocresía es siempre posible.

En segundo lugar, se puede dudar de que el hombre malo realmente emplee términos normativos de un modo genuinamente normativo, es decir, para expresar normas. Enunciados como 'Todos deben hacer $p$ ', 'Tengo la obligación de hacer $x$ ' 'Tengo el derecho a $q$ ', etc., son ambiguos y pueden expresar tanto normas como proposiciones que describen el hecho de que, en un cierto sistema jurídico, existen o son válidas normas según la cuales 'Todos deben hacer $p$ ', 'Tengo la obligación de hacer $x$ ', 'Tengo el derecho a $q$ ', etc. Así, Shapiro mismo sostiene que los juicios normativos del hombre malo expresan, no normas, sino más bien proposiciones acerca de normas de un sistema jurídico dado. Sin embargo, nada en la teoría de Hart impide adoptar la misma solución.

En tercer lugar, incluso si un hombre malo pudiese expresar juicios normativos de un modo genuinamente normativo, esto no demostraría tampoco que la solución de Hart sea incorrecta, que los silogismos HS1 y HS2 no sean válidos. Solo demostraría que las mismas conclusiones se pueden derivar de premisas diferentes, que la conexión entre las premisas y la conclusión no es un bicondicional material.

En resumen, las críticas de Shapiro contra Austin y Hart no me parecen correctas. A pesar de esto, sin embargo, hay un punto más que quisiera subrayar: creo que las soluciones que Austin y Hart dan a la PA no son tan diferentes como reputa Shapiro. Más aún, creo que las soluciones de Austin y de Hart no podrían ser diferentes, puesto que ambos son iuspositivistas. En otras palabras, creo que la aceptación del positivismo jurídico obliga a resolver la PA de un mismo modo. El verdadero problema es que la PA de Shapiro no está bien formulada.

${ }^{14}$ Hart, H.L.A., op. cit., p. 57 (72). 
En la próxima sección intentaré mostrar cómo, al introducir algunas distinciones, la paradoja se desvanece o, mejor dicho, se convierte en una cuestión no paradójica.

\section{La solución de la paradoja}

Como hemos visto, la PA se puede formular de la siguiente manera:

Huevo: Un sujeto está facultado para crear normas jurídicas únicamente si una norma existente le confiere esa potestad.

Gallina: Una norma que faculta a crear normas jurídicas existe únicamente si un sujeto con la potestad para hacerlo la ha creado.

Como dice el refrán, cuando veas una paradoja, busca una ambigüedad. De hecho, creo que si aplicamos algunas distinciones analíticas la PA desaparece, pero sigue siendo un problema, hay todavía algo que explicar.

Podemos distinguir al menos tres sentidos de 'norma existente', dos sentidos de 'norma jurídica' y dos significados principales de 'potestad de crear normas'.

En un primer sentido, una regla existe [1] en un ordenamiento jurídico, OJ, si es creada de acuerdo con todas las normas jurídicas de competencia de OJ. Llamo a este sentido de 'existencia', validez. Las normas de competencia son las normas de OJ que regulan la creación de nuevas normas. Esta clase también incluye las normas que atribuyen poder, pero no se agota en ellas ${ }^{15}$.

En un segundo sentido, una regla existe [2] en un ordenamiento jurídico, OJ, si pertenece a OJ, si se considera en general una norma jurídica de OJ, aun si no fue creada de acuerdo con todas las normas

${ }^{15}$ Así, Guastini distingue cinco subclases de normas de competencia: cf. Guastini, R., Le fonti del diritto e l'interpretazione, Milano, Giuffré, 1993; Guastini, R., "Invalidity", Ratio Juris, 7, 1994, pp. 212-226; Guastini, R., "Analysing Legislation (And Some Related Concepts)", en Annali Giurisprudenza Genova, 25, 1993-94, pp. 205-229; Guastini, R., Il diritto come linguaggio, Torino, Giappichelli, 2001. 
La paradoja de la autoridad: el positivismo jurídico...

jurídicas de competencia de OJ. Llamo este sentido de 'existencia', pertenencia ${ }^{16}$.

En consecuencia, en un primer sentido, una regla es una norma jurídica [1], es decir, es una regla de un ordenamiento jurídico, OJ, si es válida en OJ, si fue creada de acuerdo con todas las normas de OJ que gobiernan su producción y su contenido normativo. En un segundo sentido, una norma es una norma jurídica [2], esto es, es una regla de un ordenamiento jurídico, OJ, si pertenece a OJ, es decir, si existe [2] en $\mathrm{OJ}^{17}$.

En un tercer sentido, una regla existe [3] cuando fue formulada. Así, en la literatura jurídico-filosófica, la creación o producción de normas jurídicas se identifica a veces con un acto de habla (speech$a c t$ ), es decir, con la formulación de un enunciado normativo ${ }^{18}$. Dicho acto, a su vez, suele denominarse 'promulgación' ${ }^{19}$. Sin embargo, la formulación (la mera existencia de hechos, como la llama Guastini ${ }^{20}$ ) de una regla no es una condición suficiente para la existencia de una norma jurídica. Así, por ejemplo, podemos escribir la Constitución del Estado del Banano, y establecer que será obligatoria en el territorio comprendido entre la Universidad Bocconi y la calle Festa del Perdono. Pero nuestra Constitución del Estado del Banano no será una constitución jurídica, no será un conjunto de normas jurídicas. Por lo tanto, podemos omitir considerar este tercer sentido de 'existencia de normas' y analizar solo los primeros dos, a saber, la existencia como validez y la existencia como pertenencia. La distinción entre estos dos sentidos es importante porque todos los sistemas jurídicos contienen normas que no son válidas en ellos ${ }^{21}$.

16 Véase Guastini, R., "Analysing Legislation (And Some Related Concepts)”, op. cit.

17 Ídem.

18 Véase, por ejemplo, von Wright, G. H., Norm and Action. A Logical Enquiry, London, Routledge \& Kegan Paul, 1963.

${ }^{19}$ Véase, por ejemplo, Bulygin, E., "Time and Validity", en Martino, A. (ed.), Deontic Logic, Computational Linguistics, and Legal Information Systems, Amsterdam, North-Holland Publishing Co., 1982, pp. 65-81.

${ }^{20}$ Véase Guastini, R., "Analysing Legislation (And Some Related Concepts)", op. cit.

${ }^{21}$ Ídem y Guastini, R., Il diritto come linguaggio, op. cit. 
En primer lugar, en la mayoría de los ordenamientos jurídicos europeos, gobernados por constituciones rígidas, las leyes contrarias a la Constitución se consideran existentes, a pesar de su invalidez, hasta que su incompatibilidad con la Constitución sea declarada por el Tribunal Constitucional. De hecho, en la mayoría de los ordenamientos jurídicos, una regla se considera existente (es decir, perteneciente) -aunque posiblemente no válida- a condición de que haya sido emanada respetando, al menos, algunas normas de competencia, aunque no necesariamente todas ellas ${ }^{22}$. La simple pertenencia no carece de efecto jurídico: las leyes que pertenecen a un ordenamiento jurídico (aunque no sean válidas), deben ser aplicadas por los tribunales (hasta que el Tribunal Constitucional las declare derogadas, de tal manera que las normas derogadas pierden su 'pertenencia', es decir, dejan de pertenecer al ordenamiento jurídico).

En segundo lugar, las normas fundamentales de cualquier ordenamiento jurídico, como la Constitución de Italia o la Constitución de los EE. UU., no son válidas. Ello en cuanto, no fueron creadas de acuerdo con todas las normas jurídicas de competencia o, más exactamente, no fueron creadas de acuerdo con ninguna norma jurídica de competencia, pero pertenecen a los ordenamientos jurídicos que fundan.

Una reformulación del PA puede consistir en preguntarse cómo es posible que las normas fundamentales de todo ordenamiento jurídico pertenezcan a (existan [2] en) este ordenamiento, a pesar de no ser válidas. Esta pregunta es la misma que ‘Cómo es posible que la Asamblea Constituyente pueda crear normas que pertenezcan al ordenamiento jurídico, OJ, sin haber sido autorizada por otras normas pertenecientes a (o válidas en) OJ?', a saber, ‘Cómo es posible que los autores de las normas fundamentales hayan tenido la potestad de crear normas

${ }^{22}$ Como Guastini ha observado en varias oportunidades, es difícil identificar todas las condiciones necesarias y suficientes de existencia de las normas jurídicas. En principio, el cumplimiento de las normas que confieren poder parece ser una condición necesaria: pero, respecto de las normas que regulan el procedimiento de aprobación, emanación, y promulgación, la existencia parece un concepto con textura abierta (véase Guastini, R., "Analysing Legislation (And Some Related Concepts)", op. cit.; 
La paradoja de la autoridad: el positivismo jurídico...

pertenecientes a OJ, sin haber sido autorizados por otras normas pertenecientes a (o válidas en) OJ?'. Con respecto a este problema, es útil distinguir entre dos significados fundamentales de 'potestad de crear normas', o, lo que es lo mismo, 'poder normativo'.

En un primer sentido, alguien tiene la potestad de crear normas si, de hecho, crea normas o normas jurídicas. Llamo este significado de 'potestad de crear normas', poder normativo de facto. E1 poder normativo de facto es normativo en el sentido de que produce normas, pero no es normativo en el sentido de que es conferido por normas. Es un poder de facto, porque su existencia es una cuestión de hecho. La existencia de un poder normativo de facto solo puede ser determinada a posteriori examinando si, como cuestión de hecho, algunas normas han sido creadas.

En un segundo sentido, alguien tiene la potestad de crear normas si es autorizado por otras normas a crear normas. Llamo, a este significado de 'potestad de crear normas', poder normativo autorizado. Obviamente, podemos determinar si alguien tiene un poder normativo autorizado a través de un análisis a priori. Sin embargo, se necesitan algunas distinciones más.

Alguien tiene un poder normativo autorizado [1] en un ordenamiento jurídico, OJ, si ese poder es conferido por una norma que pertenece a (existe [2] en) OJ. Así, el legislador tiene un poder normativo autorizado [1] de crear normas jurídicas, porque las normas constitucionales le otorgan este poder.

Alguien tiene un poder normativo autorizado [2] en un ordenamiento jurídico, OJ, si ese poder es conferido por una norma válida en OJ. Así, en el ordenamiento jurídico italiano, los sujetos privados tienen un poder normativo autorizado [2] para crear normas contractuales porque algunas leyes (válidas) les confieren ese poder.

Por otro lado, alguien tiene un poder normativo de facto [1] en un ordenamiento jurídico, OJ, si crea normas que pertenecen a (existen [2] en) OJ.

Por último, alguien tiene un poder normativo de facto [2] en un ordenamiento jurídico, OJ, si crea normas que son válidas en OJ. Dado que una norma es válida en OJ si se crea de acuerdo con todas las nor- 
mas jurídicas de competencia de OJ, alguien tiene un poder normativo de facto [2] solo si tiene un poder normativo autorizado [1] o [2].

Ahora podemos especificar la PA de acuerdo con nuestras distinciones anteriores. Podemos ver, por ejemplo, que las siguientes especificaciones de la PA son correctas pero no paradójicas:

Huevo: Un sujeto tiene el poder normativo autorizado [1] de crear normas jurídicas (es decir, normas que pertenezcan o sean válidas en $\mathrm{OJ}$ ) únicamente si una norma existente le confiere esa potestad.

Gallina: Una norma que confiere poder normativo autorizado [1] de crear normas jurídicas existe únicamente si un sujeto con poder normativo de facto [1] la ha creado

Huevo: Un sujeto tiene el poder normativo autorizado [2] de crear normas jurídicas (es decir, normas que pertenezcan o sean válidas en OJ) solo si una norma válida le confiere ese poder.

Gallina: Una norma válida que confiere el poder normativo autorizado [2] de crear normas jurídicas existe únicamente si un sujeto con poder normativo autorizado [1] o poder normativo autorizado [2] la ha creado.

En su lugar, las siguientes versiones de la PA son paradójicas, pero falsas:

Huevo: Un sujeto tiene un poder normativo de facto [1] de crear normas jurídicas únicamente si una norma existente le confiere ese poder.

Gallina: Una norma que confiere un poder normativo de facto [1] de crear normas jurídicas existe únicamente si un sujeto con un poder normativo de facto [1] la ha creado

(es decir, alguien tiene un poder normativo de facto [1] solo si tiene un poder normativo autorizado [1]) .

Huevo: Un sujeto tiene un poder normativo de facto [2] de crear normas jurídicas únicamente si una norma existente le confiere ese poder 
La paradoja de la autoridad: el positivismo jurídico...

Gallina: Una norma que confiere un poder normativo de facto [2] de crear normas jurídicas existe únicamente si un sujeto con un poder normativo de facto [2] la ha creado

(es decir, alguien tiene un poder normativo de facto [2] solo si tiene un poder normativo autorizado [2] $)^{23}$.

Las distinciones efectuadas permiten solucionar la paradoja, pero todavía subsiste un problema. En efecto, tenemos que responder a la pregunta ‘Cómo es posible que los autores de las normas fundamentales hayan tenido el poder de crear normas que pertenecen a OJ, sin haber sido autorizados por otras normas pertenecientes a (o válidas en) OJ?'.

La respuesta positivista es simplemente que los autores de las normas fundamentales (por ejemplo, la Asamblea Constituyente) tenían un poder normativo de facto [1], y que tener un poder normativo de facto [1] es una cuestión de hecho. Alguien tiene un poder normativo de facto [1] si, como cuestión de hecho, crea normas que se consideran generalmente derecho (en un territorio dado) y, por tanto, resultan generalmente seguidas. En otras palabras, alguien tiene un poder normativo de facto [1] si, como cuestión de hecho, sus normas son efectivas porque son consideradas derecho en vigor (en un territorio determinado). Creo que esta sería la respuesta de todos los positivistas jurídicos, de Hart, de Austin, de Bentham e, incluso, de Kelsen. Más aún, creo que un positivista jurídico no podría responder de manera diferente ya que esta respuesta se sigue del concepto mismo de positivismo jurídico. Para aclarar este punto, es necesario decir algunas palabras sobre el positivismo jurídico (y sobre el modo en que lo entiende Shapiro).

${ }^{23}$ Esta versión afirma que alguien tiene el poder de crear normas que son válidas en OJ si, y solo si, este poder es conferido por una norma válida. Esta versión es falsa si admitimos que la Constitución confiere al legislador el poder de crear normas válidas y, simultáneamente, asumimos que la Constitución misma no es un conjunto de normas válidas. 


\section{E1 positivismo jurídico}

Shapiro define el positivismo jurídico como la tesis según la cual "los hechos jurídicos, en última instancia, están determinados exclusivamente por hechos sociales" 24 . Esta definición, aunque ampliamente difundida en la filosofía inglesa del derecho, no es en realidad del todo clara, ya que ni el significado de 'determinados' (o, como Shapiro a veces escribe, 'fundados'), ni el significado de 'hechos sociales' son claros. Por razones similares, la definición de iusnaturalismo ofrecida por Shapiro, como la teoría según la cual el derecho es también determinado por hechos morales, es bastante borrosa. En la filosofía continental del derecho, y en Italia en particular, podemos encontrar definiciones más perspicuas. Estoy pensando, por ejemplo, en la famosa distinción de Bobbio entre tres significados de 'positivismo jurídico': el positivismo jurídico metodológico, que consiste en asumir un enfoque neutral frente al derecho, es decir, en estudiar el derecho como es, sin valorarlo; el positivismo jurídico ideológico (o legalismo ético) que sostiene que el derecho es justo (bueno, valioso) solo porque es derecho (que una norma legal es moralmente buena solo porque es jurídicamente válida); y, por último, el positivismo jurídico teórico, que es una suma de ideologías tales como la teoría imperativa del derecho, la teoría según la cual todo sistema jurídico es coherente y completo, la tesis que sostiene que la interpretación jurídica es una actividad mecánica que consiste en conocer y declarar significados preexistentes, etc. ${ }^{25}$

De acuerdo con Bobbio, y con muchos otros autores, el núcleo del positivismo jurídico está representado por el positivismo jurídico metodológico, mientras las otras concepciones están comprometidas ideológicamente o son simplemente falsas. El positivismo jurídico metodológico no es una tesis acerca del (concepto de) derecho, es una tesis sobre la teoría del derecho. Pero, claramente, el positivismo jurídico metodológico supone un cierto concepto de derecho. Es decir,

\footnotetext{
24 Shapiro, S., op. cit., p. 55-56.

25 Véase Bobbio, N., Giusnaturalismo e giuspositivismo giuridico, Milano, edizioni di Comunità, 1965; Bobbio, N., Il positivismo giuridico, Torino, Giappichelli, 1996.
} 
La paradoja de la autoridad: el positivismo jurídico...

supone que es posible distinguir el derecho como es en realidad del derecho como debe ser. Un corolario del positivismo jurídico metodológico es precisamente la idea según la cual las normas jurídicas son jurídicamente existentes solo porque son formuladas (creadas) por seres humanos ${ }^{26}$.

Recientemente Villa ${ }^{27}$ ha propuesto una reformulación del positivismo jurídico metodológico y de su corolario, que me parece muy convincente. Villa sostiene que el concepto (y no las concepciones) del positivismo jurídico se refiere tanto a una tesis ontológica acerca del derecho cuanto a una tesis metodológica sobre el conocimiento del derecho. La tesis metodológica afirmaría que se puede hacer referencia a lo que prescribe el derecho positivo sin tener que adoptar una posición al respecto (sin tener que aceptarlo o rechazarlo). $\mathrm{La}$ tesis ontológica, por su parte, afirmaría que el derecho es el producto convencional de decisiones humanas y/o de comportamientos humanos históricamente contingentes. Me parece que, o bien la definición de positivismo jurídico formulada por Shapiro equivale (aun siendo menos precisa) a la tesis ontológica, o bien no se llega a comprender qué entiende por 'hechos sociales'.

Del mismo modo, siguiendo las distinciones precedentes, el concepto de iusnaturalismo se refiere también a una tesis metodológica y a una tesis ontológica. La tesis metodológica afirma que no es posible hacer referencia a lo que prescribe el derecho positivo sin adoptar una posición al respecto (sin aceptarlo o rechazarlo). La tesis ontológica afirma que existe un derecho natural objetivo al cual el derecho positivo debe o debería conformarse.

La tesis metodológica positivista es claramente incompatible con la tesis metodológica iusnaturalista. Pero ¿es la tesis ontológica iuspositivista también incompatible con la tesis ontológica iusnaturalista? Creo que la respuesta depende de cómo se especifique la tesis iusnatu-

${ }^{26}$ Véase, por ejemplo, Bobbio, N., Il positivismo giuridico, op. cit., p. 35; Barberis, M., Il diritto come discorso e come comportamento, Torino, Giappichelli, 1990, p. 176.

27 Véase Villa, V., 2000, "Alcune chiarificazioni concettuali sulla nozione di inclusive positivism", in Analisi e diritto 2000, p. 260-261; Villa, V., Il positivismo giuridico: metodi, teorie e giudizi di valore, Torino, Giappichelli, 2004, p. 29 y ss. 
ralista. ¿Qué pasa, según los iusnaturalistas, cuando la ley positiva es incompatible con la ley natural? A menudo, los teóricos del derecho natural responden que el derecho positivo deja de ser vinculante. El punto es: ¿'vinculante' en qué sentido? Si por 'vinculante' entendemos solo moralmente vinculante, entonces la tesis ontológica iuspositivista no es necesariamente inconsistente con la tesis ontológica iusnaturalista. La tesis ontológica iuspositivista afirma que el derecho positivo es un producto humano contingente, la tesis ontológica iusnaturalista afirma que el derecho positivo (este producto humano contingente) no es moralmente vinculante si es incompatible con la ley natural. Solo habría una inconsistencia si pensásemos que, cuando el derecho positivo viola la ley natural, el primero deja también de ser jurídicamente vinculante, es decir, deja de ser derecho.

En ambas versiones, la tesis ontológica iusnaturalista es una tesis sobre la justificación del derecho, sobre la legitimidad de la autoridad. Desde el punto de vista iusnaturalista, la pregunta '¿Cuál es el fundamento de la autoridad jurídica?’ significa ‘¿Qué justifica la autoridad jurídica? ¿Qué la legitima?'. La primera versión afirma que es la coherencia (o, mejor dicho, la no-contradicción) con la ley natural lo que justifica a la autoridad jurídica. La segunda versión añade que, cuando esta justificación está ausente, la obligatoriedad jurídica también cesa. En definitiva, la existencia del derecho positivo depende de su justificación moral ${ }^{28}$. La tesis ontológica positivista se opone a esta última versión afirmando que la existencia del derecho es independiente de su justificación moral, pero la tesis ontológica iuspositivista no responde a la pregunta sobre lo que justifica la autoridad jurídica ${ }^{29}$. Desde el punto de vista iuspositivista, la pregunta ‘Cuál es el fundamento de

${ }^{28}$ Se podría argumentar que, de acuerdo con la segunda versión, no solo el derecho en su conjunto, sino también cada norma es jurídica si es conforme a la ley natural. Es decir, una norma es válida en, o pertenece a, un ordenamiento jurídico si, y solo si, además de satisfacer las condiciones previamente identificadas (cf. sección 2), es consistente con la ley natural. Sin embargo, muchos iusnaturalistas no comparten ese punto de vista, ya que opinan que para que las normas individuales no sean jurídicas es necesario que el derecho en su conjunto tiene un alto grado de injusticia. 
La paradoja de la autoridad: el positivismo jurídico...

la autoridad jurídica?’ significa más bien ‘QQué determina la existencia de las normas jurídicas?'. La respuesta iuspositivista es que el derecho es el producto convencional de decisiones humanas y/o de comportamientos humanos históricamente contingentes. El derecho no puede más que fundarse en hechos humanos. El poder de crear normas jurídicas no puede sino derivar de un poder de facto [1], es decir, de un poder que no haya sido otorgado por ninguna otra norma jurídica. En la sección 4, veremos que esta es exactamente la solución ofrecida por Austin y Hart. Antes, sin embargo, es útil decir algo más sobre la manera en la que Shapiro concibe las tareas del iuspositivismo.

\section{III.1. Las tareas del positivismo jurídico (según Shapiro)}

Shapiro concibe al positivismo jurídico como una tesis sobre:

(a) el fundamento de la autoridad jurídica, es decir, como una respuesta a la pregunta: '¿Cuál es, en última instancia, el fundamento de la autoridad jurídica?'

(b) la identificación de las normas fundamentales de un ordenamiento jurídico determinado, es decir, como una respuesta a la pregunta: ¿Cuáles son las reglas fundamentales del ordenamiento jurídico OJ?’31;

(c) la determinación del contenido del derecho, es decir, como una respuesta a la pregunta: '¿Qué prescribe el derecho de OJ en el caso C?’32.

Creo que es difícil entender cómo el concepto iuspositivista de derecho pueda llegar a resolver todas las cuestiones planteadas por Shapiro. Claramente la tesis ontológica no puede hacerlo.

En primer lugar, como hemos visto, la tesis ontológica no responde a la pregunta ‘Cuál es el fundamento de la autoridad jurídica?' si esta es entendida como una pregunta acerca de la justificación del derecho, de la legitimidad de la autoridad.

\footnotetext{
${ }^{30}$ Véase Shapiro, S., op. cit., pp. 58-59, passim.

31 Véase ibidem, p. 60 , passim.

${ }^{32}$ Véase ibidem, pp. 56-58 y 61, passim.
} 
En segundo lugar, me parece que ningún concepto de derecho puede responder a la pregunta sobre la individuación de las normas fundamentales de un ordenamiento jurídico particular, por una razón muy trivial, a saber, el concepto de derecho tiene que distinguirse del concepto de 'derecho en vigor'. El concepto de derecho también debe abarcar el antiguo derecho romano, el derecho imaginario, en suma, todos los sistemas jurídicos que ya no están en vigor o que nunca estuvieron en vigor. El concepto de derecho nos puede ayudar a identificar las normas fundamentales, pero no va a determinar si esas son las reglas fundamentales actualmente en vigor en un ordenamiento jurídico dado ${ }^{33}$.

Por último, creo que ningún concepto de derecho puede ofrecer una solución al problema de la determinación del contenido del derecho (en un caso dado). Sobre este punto Shapiro examina la Octava Enmienda de la Constitución de los EE. UU. la cual establece que: "No se exigirá una fianza excesiva, ni se infligirán castigos crueles e inusuales". El problema es: ¿esta disposición constitucional prohíbe la pena de muerte? Shapiro señala que la interpretación de esta disposición "ha generado interminables discusiones respecto de si debe entenderse que prohíbe los castigos que realmente sean crueles o solo aquellos que quienes la redactaron y ratificaron pensaban que eran crueles" ${ }^{34}$. Según Shapiro:

La pregunta decisiva aquí es la siguiente: ¿cómo debe resolverse este desacuerdo? ¿Qué determina el contenido de la Octava Enmienda: el significado literal o la intención del constituyente (o tal vez otra cosa)? Es aquí donde el debate entre positivistas

${ }^{33}$ Sin embargo, pienso que el papel del concepto de derecho en vigor no debe ser sobrestimado, especialmente en la teoría de la adjudicación. Nunca he visto a un juez que, antes de aplicar el código civil italiano, se involucre en una investigación sociológica sobre la eficacia de la Constitución italiana. El juez simplemente asume su eficacia y es fácil para él hacerlo porque, en el país donde vive, la Constitución italiana es de hecho efectiva. Es el único conjunto de normas fundamentales eficaz. El juez emplea un concepto muy simple, no teóricamente sofisticado, de eficacia (exactamente el mismo que es empleado por todas las personas comunes). Sobre esta idea, así como sobre la distinción entre el concepto de derecho y el concepto de derecho en vigor, véase Jori, M., Il diritto inesistente, Pisa, ETS, 2010. 
La paradoja de la autoridad: el positivismo jurídico...

y iusnaturalista se torna relevante. La única manera de resolver si el significado literal o la intención del constituyente determinan el derecho constitucional de Estados Unidos es saber qué hechos determinan en última instancia el contenido de todo el derecho. Entonces, por ejemplo, si los positivistas tienen razón, la única manera de demostrar que una determinada metodología interpretativa es correcta consiste en señalar el hecho o los hechos sociales que hacen que ese sea el caso, tal vez mostrando que los tribunales habitualmente aplican una metodología y no otra. Por otra parte, si el iusnaturalista es quien está en lo correcto, entonces, la única forma de presentar la propia postura es mediante un ejercicio de filosofía moral y política. Asì, por ejemplo, uno podría defender una metodología sobre otra mostrando que existen razones vinculadas con la teoría democrática que recomiendan interpretar la Constitución de cierto $\operatorname{modo}^{35}$.

Como parece evidente, a partir de esta cita de Shapiro, ni el positivismo jurídico ni la teoría del derecho natural pueden determinar cuál es el argumento interpretativo correcto; solo pueden justificar (de manera diferente) el argumento elegido. En otras palabras, la adhesión al positivismo jurídico o a la teoría del derecho natural no impone un criterio de interpretación; solo requiere que el criterio seleccionado sea justificado de una manera determinada. Así, por un lado, no hay razón para que un positivista jurídico deba preferir el significado original y, por el otro, un teórico del derecho natural puede preferir el significado literal solo si piensa que los autores de la Constitución no estaban moralmente legitimados o bien que se equivocaron en la positivización de la ley natural. Además, la adopción del argumento interpretativo del significado literal no responde a la cuestión de si la pena de muerte está prohibida por la Constitución de los EE. UU. La pregunta sigue en pie: ¿es la pena de muerte en realidad cruel? En este caso, el argumento interpretativo subvalora la individuación del contenido de la disposi- 
ción jurídica. No es un caso inusual, creo que esto ocurre a menudo ${ }^{36}$. Así, aunque el concepto de derecho podría llevarnos a elegir entre los diferentes criterios de interpretación, no puede determinar el contenido de la ley en cada caso.

\section{El fundamento del derecho}

En la sección II he sostenido que, frente a la pregunta ‘Cómo es posible que los autores de las normas fundamentales hayan tenido el poder de crear normas que pertenecen a OJ sin haber sido autorizados por otras normas pertenecientes a (o válidas en) OJ?', el positivista jurídico tiene que responder que los autores de las normas fundamentales (por ejemplo, la Asamblea Constituyente) tenían un poder normativo de facto [1], y que tener un poder normativo de facto [1] es una cuestión de hecho. En la sección III, he tratado de mostrar cómo esta solución se sigue directamente de la tesis ontológica positivista. Para los positivistas jurídicos el derecho es el producto convencional de decisiones humanas y/o de comportamientos humanos históricamente contingentes. La existencia de las normas jurídicas no puede más que fundarse sobre hechos humanos. El poder de crear normas jurídicas no puede sino derivar de un poder de facto [1], es decir, de un poder que no fue otorgado por ninguna otra norma jurídica. Ahora es tiempo de ver que, como he adelantado, las soluciones ofrecidas por Austin y Hart a la PA se colocan bajo este enfoque y, por lo tanto, se asemejan entre ellas más de lo que Shapiro considera. No obstante, estas soluciones, tal como veremos, no quedan exentas de problemas (aunque creo que los problemas que suscitan difieren respecto a los subrayados por Shapiro).

\footnotetext{
${ }^{36}$ Podemos estar de acuerdo en que una determinada disposición debe interpretarse de acuerdo con su significado literal y todavía no estar de acuerdo acerca de cuál es su significado literal, o podemos estar de acuerdo sobre el uso del argumento teleológico y todavía no estar de acuerdo acerca de cuál es el propósito de la ley, o podemos estar de acuerdo también en la utilización de argumentos morales, pero estar en desacuerdo sobre lo que exige la moral.
} 
La paradoja de la autoridad: el positivismo jurídico...

La respuesta de Austin a la pregunta ‘Cómo es posible que los autores de las normas fundamentales hayan tenido el poder de crear normas que pertenecen a $\mathrm{OJ}$, sin haber sido autorizados por otras normas pertenecientes a (o válidas en) OJ?', es la típica respuesta iuspositivista, es decir, se trata de una cuestión de hecho. El soberano tenía el poder de crear las normas que pertenecen a OJ, ya que como cuestión de hecho, las ha formulado y los ciudadanos las han obedecido. Los hábitos de obediencia son hechos. El problema de la solución de Austin, consiste no tan solo en la incapacidad de explicar la continuidad, la persistencia y la limitación del derecho, sino, además, en que él parece pensar que solo el temor a las sanciones motiva la obediencia, y esta tesis corre el riesgo de caer en un regreso ad infinitum de las normas sancionatorias. Según Austin, los ciudadanos cumplen con las normas primarias porque piensan que es probable que si no lo hacen los jueces aplicarán las normas secundarias y los castiguen -pero ¿por qué los jueces cumplen con las normas secundarias? Sería necesario contar con otras normas (normas de tercer grado) que prescriban una sanción por la violación de las normas secundarias, otras normas (normas de cuarto grado) que establezcan una sanción para la violación de las normas de tercer grado, y así sucesivamente, ad infinitum.

Este punto merece una clarificación. Se puede opinar (como opinaba Kelsen) que la teoría del derecho no debe indagar los motivos de la eficacia del derecho, o, lo que es lo mismo, los motivos por los cuales las normas fundamentales son obedecidas, sino que debe limitarse a asumir esta eficacia, esta obediencia, como hechos dados. Esta es una posición teórica muy plausible. Pero si, por el contrario, se investigan los motivos de la eficacia y obediencia, entonces no puede sostenerse que estos se identifican simplemente con el temor a las sanciones, bajo el riesgo de un regreso ad infinitum.

También en la teoría de Hart la existencia del poder de crear normas fundamentales es una cuestión de hecho, a saber, es un poder normativo de facto [1]. La regla de reconocimiento no confiere el poder de crear las normas fundamentales: la existencia [2] de la regla de reconocimiento supone que las normas fundamentales son ya eficaces 
y aceptadas ${ }^{37}$. La regla de reconocimiento es una norma consuetudinaria, que, como cualquier norma consuetudinaria, requiere, para existir, un comportamiento general, regular, repetido a lo largo del tiempo, más la, denominada, opinio juris ac necessitatis. Así, por ejemplo, en los modernos estados de derecho la regla de reconocimiento ordena a los jueces aplicar los criterios de validez establecidos por la Constitución, pero la regla de reconocimiento en sí misma no confiere a la Asamblea Constituyente un poder autorizado para crear normas constitucionales. La existencia de la regla de reconocimiento implica que las normas constitucionales son ya eficaces (que existe ya un comportamiento regular y general conforme con ellas) y que son ya consideradas como obligatorias (ya aceptadas como estándardes jurídicos de conducta). La eficacia del ordenamiento jurídico, por ejemplo, el hecho de que en Italia los jueces siguen la Constitución italiana promulgada en el 1948, es, según la teoría de Hart, tanto un hecho como el objeto de un deber jurídico. El hecho de que exista un comportamiento regular y general junto con la creencia de que este comportamiento es jurídicamente obligatorio, genera la obligación jurídica de comportarse de esa manera. Este dispositivo puede parecer oscuro, pero tiene el mismo fundamento que cualquier norma consuetudinaria: un comportamiento general y regular, junto con la creencia general de que este comportamiento es obligatorio (o, como escribe Hart, "una combinación de conducta regular con una actitud distintiva hacia esa conducta en cuanto pauta o modelo de comportamiento"38) genera la regla según la cual esa conducta debe ser realizada.

La solución de Hart es claramente más compleja que aquellas otras que fundan las normas fundamentales solo sobre la eficacia (como la solución de Austin), pero tiene al menos dos ventajas.

En primer lugar, imaginemos escribir la Constitución del Estado del Banano e imaginemos también que nuestra Constitución es idéntica

${ }^{37}$ Sin embargo, es controvertido y, en muchos aspectos, problemático sostener que la regla de reconocimiento es una regla jurídica (una regla que pertenece al ordenamiento jurídico que funda). Este problema es muy complicado y no voy a tratarlo en este texto. 
La paradoja de la autoridad: el positivismo jurídico...

a la Constitución italiana: en ese caso, la Constitución del Estado del Banano sería eficaz. Pero, a pesar de su eficacia (entendida como mera correspondencia de hecho), no podríamos decir que la Constitución del Estado del Banano es una Constitución en vigor, un conjunto de normas fundamentales que pertenecen a (y que fundan) un ordenamiento jurídico. No lo podríamos decir porque las normas de la Constitución del Estado del Banano no serían seguidas; nadie haría lo que disponen solo porque es mandado por ellas. En términos de Hart: las normas de la Constitución del Estado del Banano no serían aceptadas. A pesar de la opinión contraria de Kelsen, la eficacia como mera correspondencia entre el comportamiento mandado por una norma y el comportamiento efectivo de los ciudadanos no es una condición suficiente para la existencia de un ordenamiento jurídico (a saber, para definir el concepto de derecho en vigor).

Una segunda ventaja de la teoría de Hart es que ella puede explicar (sin violar la ley de Hume) el hecho de que los jueces deben aplicar las normas fundamentales. Como hemos visto, en la teoría de Austin, el deber de los jueces de aplicar las normas no se puede explicar a menos que existan otras normas que castiguen a los jueces por no aplicar las normas secundarias, otras normas adicionales que castiguen a quien no aplique estas últimas normas y así sucesivamente. En la teoría de Hart, en cambio, los jueces deben aplicar los criterios de validez establecidos por la regla de reconocimiento, porque esta es exactamente una regla, una norma que se deriva de la conducta de los jueces y de su aceptación (hay un círculo, pero no vicioso).

Sin embargo, también la teoría de Hart presenta algunos problemas. Antes (I.2) hemos criticado la idea de Shapiro según la cual la teoría de Hart sería incapaz de explicar cómo un hombre malo podría expresar juicios normativos. Hemos visto que nada en la teoría de Hart impide, ni adoptar la misma solución que Shapiro (es decir, afirmar que los juicios del hombre malo expresan proposiciones acerca de normas), ni sostener, en contra de ella, que esos juicios son verdaderamente normativos, es decir, sostener que se pueden expresar juicios normativos sin aceptar las normas. Por el contrario, lo que podría parecer inverosímil en el enfoque de Hart es que el hombre malo pueda expresar juicios 
normativos y también aceptar las normas. Pero aquí hay un problema que tiene que ver con los conceptos, correlacionados, de punto de vista interno y punto de vista externo.

Por una parte, Hart caracteriza el concepto de punto de vista externo, tanto como el punto de vista de un hombre malo que "rechaz[a] sus reglas y únicamente se interés[a] en ellas porque piens[a] que la violación desencadenará, probablemente, consecuencias desagradables" ${ }^{39}$, cuanto como el punto de vista de un observador externo que registra regularidades de conducta o describe un ordenamiento jurídico dado. Pero está claro que al hombre malo no le hace falta adoptar un punto de vista externo en este último sentido. El hombre malo no es un observador, sino un jugador, aunque uno que engaña (o trata de engañar).

Por otra parte, de acuerdo con Hart, las razones para la aceptación de una regla pueden ser muy diferentes. Los participantes pueden aceptar las normas jurídicas "simplemente por deferencia a la tradición o por el deseo de identificarse con los otros o en la creencia de que la sociedad conoce mejor qué es lo que conviene a los individuos" $40 \mathrm{o}$ bien porque creen que se encuentran justificados ya sobre la base de una norma moral o cualquier otra razón prudencial. Pero si se puede aceptar una regla también sobre la base de una razón prudencial, tal como el miedo a la sanción, entonces la distinción entre la aceptación y la obediencia se desvanece y tenemos que reconocer que incluso el hombre malo puede adoptar un punto de vista interno ${ }^{41}$. Sin embargo, esta conclusión parecería insostenible, puesto que parecería que asumir un punto de vista interno respecto de una norma jurídica, como

${ }^{39}$ Ibidem, p. 90 (112).

${ }^{40}$ Hart, H. L. A., "Postscript", en The Concept of Law (1961), Second Edition. Oxford, Oxford University Press, 1994, p. 257 (35) [N. E.: no se ha consolidado aún una traducción canónica de las notas manuscritas de Hart, que Joseph Raz y Penelope Bulloch incluyeron como "Postscript" en la segunda edición de The Concept of Law (pp. 238-276). A los fines de lograr uniformidad se cita en la traducción de Rolando Tamayo y Salmorán: Hart, H. L. A., Post scriptum al concepto de derecho, México, UNAM, 2000. En paréntesis se indican las páginas correspondientes a la traducción].

${ }^{41}$ Para un análisis de los diferentes conceptos cubiertos por los términos 'aceptación' y 'punto de vista interno', véase, por ejemplo, Martin, M., The Legal Philosophy of H.L.A. Hart, Philadelphia, Temple University Press, 1987, p. 20 y ss. 
La paradoja de la autoridad: el positivismo jurídico...

"Todos deben hacer $p$ ", implica la norma "Yo, tú, todo el mundo debe hacer $p$ all things considered". Si esto es correcto, entonces se siguen dos consecuencias desagradables.

En primer lugar, como Shapiro señala, sería inexplicable cómo el hombre malo puede redescribir la ley utilizando la terminología normativa (de una manera normativa, es decir, para expresar normas y no proposiciones acerca de las normas).

En segundo lugar, como señala Schiavello ${ }^{42}$, sería difícil (por no decir imposible) fundar la autonomía del deber jurídico y distinguirlo de la obligación moral. De hecho, nuestro concepto de moral es tal que no podemos decir "Yo, tú, todo el mundo debe hacer $p$ all things considered, aunque hacer $p$ es moralmente incorrecto".

Me parece que, para resolver estos problemas, tenemos que repensar los conceptos correlacionados de punto de vista interno y punto de vista externo. Más precisamente, tenemos que admitir que incluso el hombre malo puede asumir un punto de vista interno, porque asumir un punto de vista interno no significa nada más que usar una norma como norma de conducta, evaluación y justificación (por cualquier razón, incluso por miedo a la sanción). Asumir un punto de vista interno a una norma jurídica, como "Todos deben hacer $p$ ", ni implica ni justifica la norma "Yo, tú, todo el mundo debe hacer $p$ all things considered". Solo implica y solo puede justificar la norma "Yo, tú, todo el mundo debe jurídicamente hacer $p$ ". Así, por ejemplo, como abogado, puedo decir a mi cliente que debe soportar una detención preventiva y mi juicio puede ser verdaderamente normativo, aun cuando yo crea que la detención preventiva es moralmente incorrecta. En otras palabras, asumir un punto de vista interno (o, lo que es lo mismo, aceptar una norma) solo significa usar una norma como premisa mayor de un silogismo normativo (no importa por qué). Paralelamente, también el punto de vista externo tiene que ser redefinido. Al respecto, recurrir a Chiassoni me parece muy útil ${ }^{43}$. Según Chiassoni, frente a la ley es posible optar entre dos juegos básicos, a saber, el del jugador y el del

42 Véase el artículo de Schiavello en este volumen.

${ }^{43}$ Véase el artículo de Chiassoni en este volumen. 
observador. El punto de vista externo es el punto de vista del observador. Asumir un punto de vista externo significa describir la existencia de normas, sistemas jurídicos, obligaciones, etc. El hombre malo, como todo el mundo, no asume necesariamente ni un punto de vista externo, ni un punto de vista interno; puede tomar tanto el uno como el otro, en diferentes ocasiones.

Sin embargo, algunos autores piensan que al menos algunos funcionarios tienen que aceptar la regla de reconocimiento por razones morales. No acabo de ver por qué esto es así. Me parece que, para evitar el regreso ad infinitum antes mencionado, es necesario que los jueces (o algunos de ellos) no apliquen el derecho por miedo a las sanciones, pero no es necesario que lo apliquen por razones morales.

En primer lugar, algunos autores piensan que si nadie aceptase el derecho por razones morales, entonces no habría ninguna diferencia entre el derecho y las órdenes de un bandido. Pero me parece que el experimento mental del bandido está viciado por un supuesto tácito. Este supuesto consiste en pensar que las órdenes de un bandido son siempre órdenes inmorales, y, después, se argumenta que nuestros derechos (constitucionales y democráticos) son algo diferente. ¿Por qué no tratamos de pensar las órdenes del bandido como órdenes moralmente correctas, o de preguntarnos cuál es la diferencia, por ejemplo, entre las órdenes de Jesucristo y la ley del Tercer Reich?

En segundo lugar, es necesario aclarar algunas observaciones contenidas en este ensayo que parecerían sugerir la necesidad de una aceptación moral. Dijimos que la regla de reconocimiento es una norma consuetudinaria, que existe [2] si, y solo si, hay un comportamiento regular y general conforme con ella junto con la creencia de que este comportamiento es jurídicamente obligatorio. Sobre esta base suele afirmarse que, si ningún funcionario aceptase la regla de reconocimiento por razones morales, resultaría inexplicable que no dejasen tanto de aplicarla como de creer que tienen el deber jurídico de hacerlo. Si lo hicieran, el derecho dejaría de ser tal ${ }^{44}$. Ello en cuanto las normas jurídicas solo existen si

${ }^{44}$ En realidad, creo que si solo los funcionarios dejasen de aplicar el derecho, este no dejaría de ser tal, pero comenzaría una guerra civil. 
La paradoja de la autoridad: el positivismo jurídico...

creemos en su existencia, o, mejor, las normas jurídicas dejan de existir cuando, todos juntos, dejamos de creer en ellas. Dicho de otro modo, el argumento sostiene que solo si alguno de los funcionarios acepta por razones morales se puede explicar la continuidad de la conformidad. Sin embargo, esta objeción no parece fundada, ya que ignora una evidente dificultad de hecho, es decir ¿cómo pueden los jueces, todos y al mismo tiempo, abandonar la aplicación del derecho? Sería necesaria una autoridad para coordinar a todos los jueces (en cierto sentido, no hay forma de escapar del derecho). En otras palabras, el hecho de que todos los funcionarios, al mismo tiempo, no dejan de aplicar el derecho admite también explicaciones diferentes (que no consisten en la necesaria aceptación moral por parte de, al menos, algunos funcionarios).

Por último, otros autores, como el propio Shapiro, argumentan que

"En contextos jurídicos, exigimos a las personas que paguen impuestos, se alisten al ejército, aprueben exámenes complejos ante de ejercer una profesión, y que testifiquen en juicios penales bajo la amenaza de prisión o multas graves. Solo los conceptos morales tienen el peso para plantear exigencias tan severas" 45 .

Esta observación es correcta en el sentido de que el derecho es tan importante que debe estar moralmente justificado. Nosotros (moralmente) tenemos que obedecer al derecho solo si pensamos que está moralmente justificado, pero esto no implica que, de hecho, el derecho esté moralmente justificado.

Como comentario final, creo que las consideraciones anteriores también muestran por qué la objeción de Dworkin fundada en el ejemplo del vegetariano, no resulta correcta. Dworkin afirma que la existencia de una obligación (moral) es independiente de la existencia de una norma social. Así, si yo soy la única vegetariana en el mundo, puedo pensar que comer carne es malo, que no se debe comer carne, aun cuando nadie más que yo lo creyese, aun si no existiese una norma social que prohíba comer carne. Sin embargo, aunque se pueda admitir que hay obligaciones morales sin reglas sociales, no puede admitirse que haya una obligación de aplicar algunos criterios de validez si no hay una regla

${ }^{45}$ Shapiro, S., op. cit., p. 155. 
social de reconocimiento. Yo podría tener la creencia de estar obligada a reconocer como válidas solo las normas que se conformasen a los criterios de validez de la Constitución del Estado del Banano, pero si así lo creyera, sería sin dudas considerada una loca por los demás.

\section{La solución de Shapiro a la paradoja de la autoridad}

La teoría de Shapiro

sostiene que un órgano tiene autoridad jurídica en un sistema jurídico en particular cuando se satisfacen dos condiciones: (1) el plan maestro del sistema autoriza a ese órgano a planificar para otros, y (2) los miembros de la comunidad en general obedecen a quienes gozan de esa autorización. Por lo tanto, la autoridad jurídica será posible solo si es posible que estas dos condiciones tengan lugar ${ }^{46}$.

En cuanto al primer requisito, Shapiro sostiene que los funcionarios públicos

tienen la potestad de adoptar el plan compartido [i.e. el plan maestro] que establece estas reglas fundamentales en virtud de las normas de la racionalidad instrumental. Puesto que estas normas que confieren la capacidad racional de planificar no son planes ellas mismas, no han sido creadas por ninguna otra autoridad. Existen solo en virtud de que son principios racionalmente válidos ${ }^{47}$.

En cuanto al segundo requisito, Shapiro afirma que

Los miembros del grupo pueden aceptar una política general de obedecer al derecho o considerar que quienes tienen autoridad están legitimados moralmente. En tales casos, la adopción de planes por parte de los funcionarios públicos generará la exigen-

\footnotetext{
46 Ibidem, p. 228.
}

98

${ }^{47}$ Ibidem, pp. 228-229. 
La paradoja de la autoridad: el positivismo jurídico...

cia racional de que esos individuos cumplan. Incluso cuando los miembros del grupo no están predispuestos a conformarse al derecho, el compromiso de los funcionarios de llevar a cabo parte del plan compartido que ordenan un castigo en caso de desobediencia puede ser suficiente para motivar la obediencia de los ciudadanos comunes ${ }^{48}$.

Voy a dedicar solo unas pocas palabras a la solución de Shapiro a la PA porque ya ha sido examinada en otros ensayos de este mismo volumen, sobre todo en el de Pierluigi Chiassoni.

Contra Chiassoni, creo que la solución de Shapiro es una solución iuspositivista. Lo creo en el sentido de que, según la teoría de los planes, el derecho también es el producto contingente de conductas y decisiones humanas. Como hemos visto antes, el problema planteado por la PA es "¿Cómo es posible que las normas fundamentales de todo ordenamiento jurídico, OJ, pertenezcan a ese ordenamiento sin ser válidas?" o, lo que es lo mismo, “¿Cómo se es posible que los autores de las normas fundamentales hayan tenido el poder de crear normas que pertenecen a OJ sin haber sido autorizados por otras normas pertenecientes a (o válidas en) OJ?". En términos de Shapiro estas preguntas son equivalentes a "¿Qué autorizó a los autores del plan maestro?" o, mejor, "¿Cómo es posible que el plan maestro pueda crear normas que pertenecen a OJ, sin estar autorizado por otras normas que pertenecen a (o son válidas en) OJ?". La respuesta de Shapiro es que esta es una cuestión de hechos: "los sistemas jurídicos son posibles porque ciertos estados de cosas son alcanzables"49.

En realidad se podría objetar que -según Shapiro- el plan maestro está autorizado por las normas de la racionalidad instrumental (las cuales, sin embargo, no autorizan a los autores del plan maestro, sino a los funcionarios, que adoptan el plan maestro y no lo escriben). Pero me parece que las normas de la racionalidad instrumental no son, en

48 Ibidem, p. 229.

49 Por otra parte, Shapiro no se pregunta por la legitimidad moral del derecho, especificando que "no hay razón para pensar que el plan maestro de todo posible sistema jurídico vaya a ser moralmente legítimo" (idem). 
sentido estricto, normas; no son guías para las acciones, no motivan comportamientos. Por un lado, las normas de la racionalidad instrumental parecen normas técnicas, o, mejor dicho, definiciones de lo que se considera racional. El hecho de que hacer algo sea racional no implica que deba hacerlo, a menos que yo quiera ser racional. Por otro lado, la capacidad de adoptar un plan es un hecho, una habilidad humana.

Creo que uno de los problemas de la teoría de Shapiro es que no puede explicar el deber jurídico de los jueces de aplicar el plan maestro. Los funcionarios pueden adoptar el plan maestro, pero no tienen el deber de hacerlo. En realidad, los funcionarios no tienen el deber jurídico de aplicar el plan maestro, aunque lo hayan adoptado. Como escribe Shapiro,

Un funcionario que acepta su posición en una estructura de autoridad será racionalmente criticable si desobedece a sus superiores, omite completar sus órdenes a fin de tomar las medidas necesarias para satisfacer sus exigencias, adopta planes inconsistentes con estas órdenes o las reconsidera sin una buena razón para ello ${ }^{50}$.

Pero ser racionalmente criticable no significa ser jurídicamente criticable. Alguien es racionalmente criticable si viola las reglas de la racionalidad que, por un lado, no son normas jurídicas, y, por el otro, no son normas en sentido estricto.

En la teoría de Hart (tal como fue reconstruida en la sección IV), la aceptación de un juez no es una condición necesaria para que el juez en cuestión tenga el deber jurídico de hacer $p$ (por ejemplo, de aplicar un dado criterio de validez). Si la regla de reconocimiento existe, si los demás jueces la aceptan, entonces es cierto que existe el deber de hacer lo que esta regla requiere. En su lugar, me parece que en la teoría de Shapiro la adopción por el juez del plan maestro es una condición necesaria para que este mismo juez tenga el deber racional de hacer $p$ (es decir, lo que requiere el plan maestro). Pero, dado que los deberes 
racionales no son deberes normativos y jurídicos, el deber jurídico de los jueces de aplicar el plan maestro permanece inexplicado.

Por otra parte, la tesis de Shapiro no explica tampoco los enunciados jurídicos normativos, es decir, no explica el significado de frases como "Todos los jueces deben respetar la Constitución italiana", "Tú tienes el deber jurídico de hacer $p$ ", etc., si los entendemos como auténticos juicios normativos. Según Shapiro, todos los enunciados anteriores son descriptivos y significan "Según el punto de vista de un ordenamiento jurídico dado, cada juez moralmente debe respetar la Constitución italiana", "Según el punto de vista de un ordenamiento jurídico dado, tú tienes el deber moral de hacer $p$ ", etc. Estos juicios son normativos si, y solo si, el hablante comparte la teoría moral del ordenamiento jurídico en cuestión, es decir, si, y solo si, expresan juicios morales. Me parece que Shapiro se torna pasible de las mismas críticas que él ha dirigido contra Austin, en cuanto la tesis de Shapiro no es capaz de hacer inteligible el pensamiento jurídico. Shapiro rechaza la autonomía del razonamiento jurídico y niega la existencia de una pluralidad de sistemas normativos. La normatividad parece ser solamente una cuestión moral.

\section{Bibliografía}

Barberis, M., Il diritto come discorso e come comportamento, Torino, Giappichelli, 1990.

Bobbio, N., Giusnaturalismo e giuspositivismo giuridico, Milano, Edizioni di Comunità, 1965.

Bobbio, N., Il positivismo giuridico, Torino, Giappichelli, 1996.

Bulygin, E., "Time and Validity", en A.A. Martino (ed.), Deontic Logic, Computational Linguistics, and Legal Information Systems, Amsterdam, North-Holland Publishing Co., 1982, pp. 65-81.

Chiassoni, P., "Una mirada desde el realismo analítico: vieja metafísica, señorío de las verdades obvias y positivismo sospechoso" en este volumen. 
Ferrer Beltrán, J. y Ratti, G. B., "Una mirada positivista a los desacuerdos teóricos" en este volumen

Guastini, R., Le fonti del diritto e l'interpretazione, Milano, Giuffré, 1993. Guastini, R., "Analysing Legislation (And Some Related Concepts)", en Annali Giurisprudenza Genova, 25, 1993-94, pp. 205-229.

Guastini, R., "Invalidity", Ratio Juris, 7, 1994, pp. 212-226.

Guastini, R., Il diritto come linguaggio, Torino, Giappichelli, 2001.

Hart, H. L. A. (1961), The Concept of Law, Second Edition, Oxford, Oxford University Press, 1994.

Hart, H. L. A., El concepto de derecho, Buenos Aires, Abeledo-Perrot, 1963, traducción de Genaro Carrió.

Hart, H. L. A., Post scriptum al concepto de derecho, México, UNAM, 2000, traducción de Rolando Tamayo y Salmorán.

Jori, Mario, Il diritto inesistente, Pisa, ETS, 2010.

Martin, M., The Legal Philosophy of H.L.A. Hart, Philadelphia, Temple University Press, 1987.

Schiavello, A., "Norma de reconocimiento, convención y obligación. Aquello que Shapiro todavía puede aprender de los errores de Hart", en este volumen.

Shapiro, S., Legalidad, Madrid-Barcelona, Marcial Pons, 2014, traducción de Papayannis, D. M. y Ramírez Ludeña, L.

Villa, V., "Alcune chiarificazioni concettuali sulla nozione di inclusive positivism", en Analisi e diritto, 2000, pp. 255-288.

Villa, V., Il positivismo giuridico: metodi, teorie e giudizi di valore, Torino, Giappichelli, 2004.

Von Wright, G. H., Norm and Action. A Logical Enquiry, London, Routledge \& Kegan Paul, 1963. 